\title{
XEROSTOMIA: RECOGNITION, DENTAL IMPLICATION AND ITS MANAGEMENT
}

Jameel Ahmad Saib, Khan Sameena.

1. Assistant Professor, Consultanting Prosthodontist, Implant and Maxillo-facial Prosthesist. Department of Dental Sciences, Narayan Medical College and hospitals. Jamuhar,Sasarm .Rohtas.(Bihar)

2. Practice, Consulting Dentist and Oral \& Maxillofacial Surgeon.

\section{CORRESPONDING AUTHOR}

Dr. AHMAD SAIB JAMEEL

4/1360. 'AL-HASNA',

Near Badi Masjid, Sir Syed Nagar,

Aligarh- 202002. UP, India.

E-mail: dr_dosmile@yahoo.co.in, ahmadsaib11@gmail.com

Ph: 0091 9412594147, 07417620934

ABSTRACT: Xerostomia commonly called as dry mouth, is the subjective feeling of oral dryness. It is not a diagnosis, but a symptom with multiple possible causes. Although dry mouth is most frequently associated with altered salivary gland function, there are other etiologies for this condition. The most frequent cause of dry mouth complaints is the use of certain drugs, depression or psychological conditions, systemic diseases, radiotherapy etc. It is important to perform a complete evaluation of the patient with dry mouth, to determine the possible cause of the xerostomia so that appropriate management can be instituted in a timely manner. The patient with xerostomia who has salivary gland hypo- function is at risk for many oral complications and it is critical to institute appropriate preventive measures. The dentist and dental hygienist has an important role in recognition and management of the patient with xerostomia. Xerostomia may also be a consequence of systemic disease and early recognition may aid in their prompt treatment. Management may be viewed as a series of goals: to provide symptomatic relief of dryness complaints; to institute appropriate preventive measures; to treat any ongoing oral problems; if salivary function is decreased, to stimulate remaining function; and to address the underlying disease process.

KEYWORDS: Xerostomia, Saliva, Recognition, Dental implications, Management

INTRODUCTION: Xerostomia is defined as dry mouth resulting from reduced or absent salivary flow. Saliva plays a critical role in the preservation of oropharyngeal health and persistent dry mouth and salivary dysfunction can impair a person's quality of life. Reduced salivary flow is common in the elderly, and is associated with a variety of factors:

- therapeutic head and neck irradiation

- Sjogren's syndrome

- HIV, diabetes, renal failure

- Psychosomatic disorder

- and pharmacotherapy.

Oral manifestations of hypo salivation typically include glossitis, mucositis, angular cheilosis, dysgeusia, difficulty in chewing and swallowing, 
EPIDEMIOLOGY AND ETIOLOGY OF XEROSTOMIA: Xerostomia is a common oral concern for many patients. It is estimated that up to 10 percent of the general population experiences persistent oral dryness. ${ }^{1,2}$ Xerostomia is more frequent with increasing age, and over $25 \%$ of elders complain of daily dryness. ${ }^{3}$ That percentage is even higher in institutionalized elders. However, it should be recognized that xerostomia is not a result of aging per se, and should not be dismissed as such. Salivary function in healthy, non-medicated elders does not vary significantly from younger individuals. It is believed that the increased frequency of dryness complaints with aging is a result of systemic disease and medication use, both more common in elders and both associated with xerostomia. ${ }^{4}$ As noted earlier, there are non-salivary causes of xerostomia. These include conditions in which there are alterations of oral mucosal sensation or central cognitive changes, such as following a cerebral vascular accident (stroke). Dryness has been associated with depression, even in the absence of recognizable changes in salivary function.

The most common causes of xerostomia, however, are conditions or circumstances that result in alterations in salivary gland function, either quantitative or qualitative. ${ }^{1}$

a) The most frequent cause of dry mouth complaints is the use of prescription drugs. There are hundreds of pharmaceuticals that have xerostomia as a side effect.5, 6 The salivary glands are stimulated strongly by cholinergic agents. Therefore, anti-cholinergic medications, such as antihistamines, are most likely to cause decreased salivary output and xerostomia. ${ }^{5}$ However, many other classes of medications, including sedatives, antipsychotics, antidepressants and diuretics are associated with xerostomia.

b) Interestingly, many herbal preparations may induce complaints of oral dryness, and patients should always be questioned about non-prescription drug use. ${ }^{7}$ Examples of commonly used preparations include members of the Labiatae family (specifically all the salvias), capsicum, garlic, Ginkgo biloba, and St. John's Wort (Hypercicum perforatum). Herbal preparations with strong diuretic properties, such as stinging nettle (Urtica dioica) and dandelion (Taraxacum officinale) also may lead to complaints of dry mouth. Caffeine is well recognized as a cause of xerostomia. ${ }^{8}$

c) There is also medical therapies that result in xerostomia. Radiotherapy to the head and neck region that includes the salivary glands in the treatment fields can lead to significant and persistent dry mouth. An expected complication at exposure levels above $5200 \mathrm{cGy} .{ }^{\mathbf{9}, 10}$ Systemically administered radionuclide's, such as 131 I for treatment of thyroid cancer, are a recognized cause of dry mouth, particularly when re-treatment is required.11

d) Another major cause of xerostomia is systemic disease. There are a large number of conditions that can affect salivary gland function and lead to complaints of dryness(Table I). ${ }^{12}$ The most prominent of the connective tissue diseases is Jorgen's syndrome, an autoimmune condition . Xerostomia is one of the hallmark symptoms in Jorgen's syndrome, experienced by over 95 percent of patients. ${ }^{13}$ Patients experience a number of dryness complaints in addition to xerostomia. These include eye, nose, throat, skin and vaginal dryness. Since complaints of oral dryness typically do not appear until salivary function has been reduced by approximately 50 percent. ${ }^{14}$ Hence more than a single gland must be affected for xerostomia to manifest. This emphasizes that dryness resulting from salivary dysfunction is usually indicative of systemic involvement. 
Table I.

Systemic Conditions that may have Xerostomia as a Symptom 12

A. Autoimmune connective tissue disorders

- Sjögren's syndrome, primary and secondary

B. Granulomatous diseases

- sarcoidosis, tuberculosis

C. Endocrine imbalance

- Diabetes (poorly controlled)

- Thyroid disease

- hypo- and hyper-thyroidism

- Amyloidosis

D. Cystic fibrosis

E. Graft-versus-host disease

F. Bell's palsy

G. Human immunodeficiency virus infection

H. Eating disorders and malnutrition

- anorexia, bulimia, dehydration

DIAGNOSIS OF XEROSTOMIA: It is critical that oral health care practitioners recognize xerostomia and salivary gland dysfunction in their patients, as saliva plays an essential role in protection of oral hard and soft tissues and support of oral functions. ${ }^{15}$ One should follow a systematic assessment approach to the patient with xerostomia:

1) Listen for and elicit symptomatic complaints,

2) Examine for oral signs of salivary gland dysfunction and

3) Evaluate salivary gland function.

Additionally, patients may report difficulty in chewing, swallowing, or speaking. A need to drink fluids to help swallow while eating or an inability to swallow dry foods is also common in such patient. Pain is often reported, and the oral mucosa may be sensitive to spicy or coarse foods, which may limit the patient's diet and enjoyment of meals. Removable denture wearers commonly report problems with retention and mucosal damage due to lack of lubrication. Eye, throat, nasal, skin or vaginal dryness, in addition to xerostomia, may be indicative of a systemic condition, such as Sjögren's syndrome.

\section{CLINICAL ASSESMENT OF XEROSTOMIA:}

On examination, the patient with xerostomia due to reduced salivary gland function usually has obvious signs of mucosal dryness. ${ }^{1}$

- The lips may be cracked, peeling and atrophic.

- The buccal mucosa pale and corrugated in appearance.

- The tongue smooth and reddened with loss of papillation (Figure1).

- The oral mucosa may appear reddened, thinner and more fragile.

- There is often a marked increase in erosion and dental caries, particularly at the gingival margin, and even cusp tip involvement.

- The decay may be rapid and progressive even in the presence of excellent oral hygiene (Figure 2). One should consider whether the caries history and current condition are consistent with the patient's oral hygiene.

- Candidiasis, most frequently of the erythematous form. 
- The salivary glands should be examined for enlargement, pain, changes in texture, and to determine if saliva can be expressed from the main excretory ducts.

- The saliva should be clear, watery and copious. Any change or cloudy exudates may be a sign of bacterial infection, although some patients with very low salivary output will have opaque secretions that are sterile. In addition to these physical effects on the oral cavity, a patient's quality of life is severely impacted.16,17

\section{LABORATORY ASSESMENT AND OTHER DIAGNOSTIC AIDS:}

Since adequate salivary function is important to support alimentation and communication, salivary gland dysfunction and xerostomia may adversely affect some of our most critical activities of daily living. Salivary gland function should be assessed in the patient with xerostomia. Using a well-defined, standardized, and clearly documented procedure for collection will allow meaningful comparisons to published material and with repeat measures in an individual over time. The composition of the collected saliva may be analyzed (sialochemistry). Over 60 components have been identified in saliva. ${ }^{18}$ There is increasing interest in salivary diagnostics for systemic and oral diseases. ${ }^{19}$ Saliva is used currently for determining blood alcohol levels, smoking and drugs of abuse as well as for HIV testing and detection of exposure to other microbes.

Using newer genomic and proteomic techniques, researchers have begun to identify possible salivary biomarkers for a number of oral and systemic conditions.

- This is a rapidly expanding area of investigation. Salivary function and gland integrity also can be examined with Technetium (Tc) pertechnetate scintiscanning. Uptake of intravenously injected $99 \mathrm{mTc}$ tracer from the blood into the glands and subsequent secretion into the oral cavity can be monitored with a gamma camera.

- Tumors and structural alterations in the salivary glands can be visualized with sialography, ultrasonography, MRI or CT imaging.20 These techniques are useful to detect salivary stones (sialoliths), duct blockage, constriction or damage, tumors and cysts. These are not functional measures, but are useful for diagnosis of salivary dysfunctions. Biopsy of the salivary glands can be done as well. Laboratory studies may be helpful in the diagnosis of Sjögren's syndrome. Using a systematic evaluation approach, the cause of dry mouth complaints can be identified in about 80 percent of patients. The remainder of patients is characterized as having idiopathic xerostomia, but still requires palliative care for symptomatic relief.

\section{DENTAL PROFESSIONAL'S ROLE AND MANAGEMENT OF XEROSTOMIA:}

Management of xerostomia is a team process, involving the dentist, the dental hygienist and the patient's physicians. Effective communication is essential for optimal patient care. The dentist and dental hygienist have an important role in recognition and management of the patient with xerostomia. With careful attention and regular follow-up, most patients will achieve increased in oral comfort and decreased oral complications can be minimized. Management may be directed to achieve series of goals:

1) to provide symptomatic relief of dryness complaints,

2) to institute appropriate preventive measures,

3) to treat any ongoing oral problems,

4) if salivary function is decreased, to stimulate remaining function and

5) to address the underlying disease process. 
Management starts with methods to relieve symptoms. Frequent sips of water will help to relieve dryness, ease swallowing, hydrate tissues and cleanse the mouth. As noted earlier, many patients with dry mouth carry water with them at all times, and this should be encouraged. Oral rinses and mouthwashes, gels, sprays and artificial saliva's are frequently used and may reduce discomfort and improve function transiently. The dental hygienist should advise the patient to use oral care products that have been specifically formulated for a dry mouth; that is, without alcohol, using only mild detergents and flavorings, having a neutral or alkaline $\mathrm{pH}$, containing no sugar and with added lubricants. The use of chewing gums, lozenges, candies or mints may provide symptomatic relief by stimulating salivation, but they must be sugar-free and nonacidic. Products containing xylitol as a sweetening agent should be encouraged, as xylitol has been shown to have anti-caries properties. For dry lips, a hydrating cream or ointment may help relieve symptoms. Use of products with Aloe Vera or vitamin E should be encouraged. Highly flavored toothpastes should be avoided because they may irritate the dry mucosa and cause pain or sensitivity. A diet that contains moisture-rich foods and not hot or spicy foods may be more comfortable for the patient. Alcohol and caffeine may produce further drying and should be avoided or used only in limited quantities. In addition to its other negative health consequences, smoking may be drying and irritating to the mucosa and should be avoided. An increase in environmental humidity is exceedingly important. Patients, especially in the wintertime, often experience a worsening of their symptoms. Use of room humidifiers, particularly at night, may improve discomfort markedly.

Preventive measures are necessary to minimize oral hard and soft-tissue damage. Patients should be seen by the dental hygienist on a frequent recall schedule of at least three times per year, until symptoms are minimized and dental caries is controlled. They must be instructed in and practice vigilant home oral hygiene. Patients should brush with fluoride toothpaste after each meal. At a minimum, rinsing the mouth immediately after eating to remove food debris is beneficial. Advice on diet is critical. Hygienists must stress the importance of extremely low sugar intake, the use of sugar-free substitutes and the avoidance of sticky, Carbohydrate-rich foods to minimize the caries risk. Patients should also be aware of the increased risk of tooth wear. Patients must be careful not to consume acidic foods and beverages in excess (e.g. carbonated sodas) in an effort to relieve symptoms. Remineralizing mouth rinses are now available and their use should be encouraged. Patients with dry mouth have an increased risk of fungal infections. If a fungal infection is present, appropriate antifungal treatments should be started. Due to the salivary dysfunction, treatment may be prolonged and re-treatment is frequently required. A therapeutic agent should be selected that does not contain sugar as a flavoring agent, as is common in many antifungal lozenges. Nystatin rinses may be prepared by a pharmacist from powder without the added flavoring ingredient(s).

Dental caries management is based on the severity of salivary dysfunction and the observed caries rate. All patients with xerostomia should use some supplemental fluoride, in addition to a fluoride containing toothpaste. Selection of the type, dose and frequency of application should be made in consultation between the dentist, the dental hygienist and the patient. Often a combination of office-applied and home-based fluoride treatments is optimal. Stimulating salivary gland flow is effective for relief of dryness symptoms and provides the myriad benefits of natural saliva. Chewing will stimulate salivary flow effectively, as will sour tastes. The combination of chewing and taste, as provided by gums or mints, can be very effective in relieving symptoms for patients who have remaining salivary function. As noted, however, patients must be told not to use products containing sugar as a sweetener. Electrical stimulation 
of the salivary glands has been attempted, but application is a challenge and the results have been modest at best. Presently, newer devices are being investigated and show promise for the future. ${ }^{21}$

Systemic drugs, termed secretogogues, may be prescribed. The Food and Drug Administration (FDA) has approved two medications for the relief of dry mouth symptoms in Sjögren's syndrome: pilocarpine (Salagen () and cevimeline (Evoxac ()$^{22-23}$ These medications are parasympathomimetic, muscarinic agonists, which induce a transient increase in salivary output and statistically significant improvement in complaints of oral dryness. Common side effects of both medications include sweating, flushing, urinary incontinence and gastrointestinal discomfort. While rarely severe or serious, side effects are frequent, which may limit the usefulness of these agents in some individuals. Parasympathomimetics are contraindicated in patients with uncontrolled asthma, narrow-angle glaucoma, and acute iritis and should be used with caution in patients with significant cardiovascular disease, Parkinson disease, asthma and chronic obstructive pulmonary disease. Pilocarpine is recommended at a dosage of $5 \mathrm{mg}$ up to four times daily, while cevimeline is prescribed at $30 \mathrm{mg}$ three times daily. These medications are widely used and provide significant relief of dryness complaints in many patients. Pilocarpine has also been approved for treatment of xerostomia related to head and neck radiotherapy.

\section{NEWER APPROACH:}

A number of different approaches have been attempted to address the underlying disease process and therefore improve salivary gland dysfunction, particularly in Sjögren's syndrome. Current studies are targeting specific biologic pathways to modify the underlying autoimmunity.

- Very low dose interferon-alpha, 150 or 450 IU once or three times a day orally, has been studied in primary Sjögren's syndrome. Use of 150 IU interferon-alpha three times daily resulted in increased stimulated whole salivary flow rates at 12 weeks compared to placebo. ${ }^{24} \mathrm{~A}$ subsequent phase 3 trial at this dose found increased unstimulated salivary function compared to placebo at 24 weeks.

- At present, there is interest in modulation of B-cell activation. As there is welldocumented B-cell hyperreactivity in Sjögren's syndrome, this approach may have an impact on the underlying disease. Rituximab is a humanized monoclonal antibody that binds specifically to the CD20 antigen, which is present on B lymphocytes and is involved in cell activation. In an open-label study, improvements in symptoms of dry mouth and salivary gland function were demonstrated. ${ }^{25}$ Although these results are promising, a randomized controlled trial is necessary before recommendation of this treatment option for Sjögren's syndrome. A recent open-label trial looked at an antiCD22 monoclonal antibody and reported very promising results in Sjögren's syndrome. ${ }^{26}$ Further studies are anticipated.

Management of xerostomia requires a team approach. The dentist and dental hygienist should work together closely to minimize the patient's dryness complaints and to preserve and protect the oral tissues. Patient education is key. The dental team needs to explain the oral sequel of dry mouth and the problems and issues patients may encounter in their daily lives. The oral condition needs to be assessed and monitored closely, and issues such as oral hygiene care and diet should be emphasized. Consultation with the patient's physician is indicated if, for example, the xerostomia appears to be related to medication use. In this case, discussion of alternative medications or dosing regimens to minimize xerostomia is indicated. If systemic 
disease is suspected as the underlying cause of salivary dysfunction, patients should be referred to their physician or an appropriate specialist without delay. Ongoing review and monitoring of the patient's oral and general health are important for optimal management.

SUMMARY AND CONCLUSION: Awareness and recognition of xerostomia are essential in order to help patients minimize dryness symptoms, to institute preventive measures and to limit oral complications. The dental hygienist has the opportunity to ask every patient if they are experiencing dry mouth. In particular, complaints of dryness while eating, or difficulty swallowing dry foods, or the necessity of using liquids to ease swallowing are important clues that salivary function may be impaired. As part of a routine oral examination, one should examine the oral cavity carefully for signs of salivary gland dysfunction. Findings such as an increase in caries activity, mucosal alterations, infection or salivary gland enlargement may indicate salivary dysfunction. Evaluation should be conducted proactively at each patient visit. Early recognition will minimize damage and dysfunction and allow appropriate management to begin. Although the salivary dysfunction may be irreversible, preventive measures and conservative treatments can avoid or limit mucosal breakdown, infections and permanent damage to teeth. Adequate symptomatic relief is possible with local palliative and systemic measures in many patients. Appropriate management of symptoms and increasing saliva output may help patients feel more comfortable and improve their quality of life.

\section{REFERENCES:}

1. 1.Guggenheimer J, Moore PA. Xerostomia: etiology, recognition and treatment. J Am Dent Assoc 2003; 134(1): 61-9.

2. Atkinson JC, Grisius M, Massey W. Salivary hypo function and xerostomia: diagnosis and treatment. Dent Clin North Am. 2005; 49(2): 309-26.

3. Orellana MF, Lagravère MO, Boychuk DG, et al. Prevalence of xerostomia in populationbased samples: a systematic review. J Public Health Dent 2006; 66(2): 152-8.

4. Ship JA, Pillemer SR, Baum BJ. Xerostomia and the geriatric patient. J Am Geriatr Soc 2002; 50(3): 535-43.

5. Ciancio SG. Medications' impact on oral health. J Am Dent Assoc 2004; 135(10): 1440-8.

6. Sreebny LM. Saliva in health and disease: an appraisal and update. Int Dent J 2000; 50(3): 140-61.

7. Abebe W. An overview of herbal supplement utilization with particular emphasis on possible interactions with dental drugs and oral manifestations. J Dent Hyg 2003; 77(1): 37-46.

8. Sreebny LM, Schwartz SS. A reference guide to drugs and dry mouth-2nd edition. Gerodontology. 1997; 14(1): 33-47.

9. Sciubba JJ, Goldenberg D. Oral complications of radiotherapy. Lancet Oncol 2006; 7(2): 175-83.

10. Dirix P, Nuyts $S$, Van den Bogaert W. Radiation-induced xerostomia in patients with head and neck cancer: a literature review. Cancer 2006; 107(11): 2525-34.

11. Mandel SJ, Mandel L. Radioactive iodine and the salivary glands. Thyroid 2003; 13(3): 265-71. 
12. von Bültzingslöwen I, Sollecito TP, Fox PC, et al. Salivary dysfunction associated with systemic diseases: systematic review and clinical management recommendations. Oral Surg Oral Med Oral Pathol Oral Radiol Endod 2007;103 Suppl: S57.e1-15.

13. Kassan SS, Moutsopoulos HM. Clinical manifestations and early diagnosis of Sjögren syndrome. Arch Intern Med 2004; 164(12):1275-84.

14. Dawes C. Physiological factors affecting salivary flow rate, oral sugar clearance, and the sensation of dry mouth in man. J Dent Res 1987; 66 Spec No: 648-53.

15. Dodds MW, Johnson DA, Yeh CK. Health benefits of saliva: a review. J Dent 2005; 33(3): 223-33.

16. Ikebe K, Matsuda K, Morii K, et al. Impact of dry mouth and hyposalivation on oral health-related quality of life of elderly Japanese. Oral Surg Oral Med Oral Pathol Oral Radiol Endod 2007; 103(2): 216-22.

17. Perno Goldie M. Xerostomia and quality of life. Int J Dent Hyg 2007; 5(1): 60-1.

18. Pesce MA, Spitalnik SL. Saliva and the clinical pathology laboratory. Ann NY Acad Sci 2007; 1098: 192-9.

19. Chiappin S, Antonelli G, Gatti R, De Palo EF. Saliva specimen: a new laboratory tool for diagnostic and basic investigation. Clin Chim Acta 2007; 383(1-2): 30-40.

20. Thoeny HC. Imaging of salivary gland tumours. Cancer Imaging 2007; 7: 52-62.

21. Strietzel FP, Martín-Granizo R, Fedele $S$, et al. Electrostimulating device in the management of xerostomia. Oral Dis 2007; 13(2):206-13.

22. Vivino FB, Al-Hashimi I, Khan Z, et al. Pilocarpine tablets for the treatment of dry mouth and dry eye symptoms in patients with Sjögren syndrome: A randomized placebocontrolled fixed-dose multicenter trial. Arch Intern Med 1999; 159: 174-181.

23. Petrone D, Condemi JJ, Fife R, et al. A double-blind, randomized,placebo-controlled study of cevimeline in Sjogren's syndrome patients with xerostomia and keratoconjunctivitis sicca. Arthritis Rheum 2002; 46(3): 748-54.

24. Ship JA, Fox PC, Michalek JE, et al. Treatment of primary Sjögren's syndrome with lowdose natural human interferon-alpha administered by the oral mucosal route: a phase II clinical trial. IFN protocol study group. J Interferon Cytokine Res 1999; 19:94351.

25. Pijpe J, van Imhoff GW, Spijkervet FK, et al. Rituximab treatment in patients with primary Sjogren's syndrome: an open-label phase II study. Arthritis Rheum 2005; 52(9): 2740-50.

26. Steinfeld SD, Tant L, Burmester GR, et al. Epratuzumab (humanized anti-CD22 antibody) in primary Sjögren's syndrome: an open-label phase I/II study. Arthritis Res Ther 2006; 8(4): R129. 


\section{ORIGINAL ARTICLE}

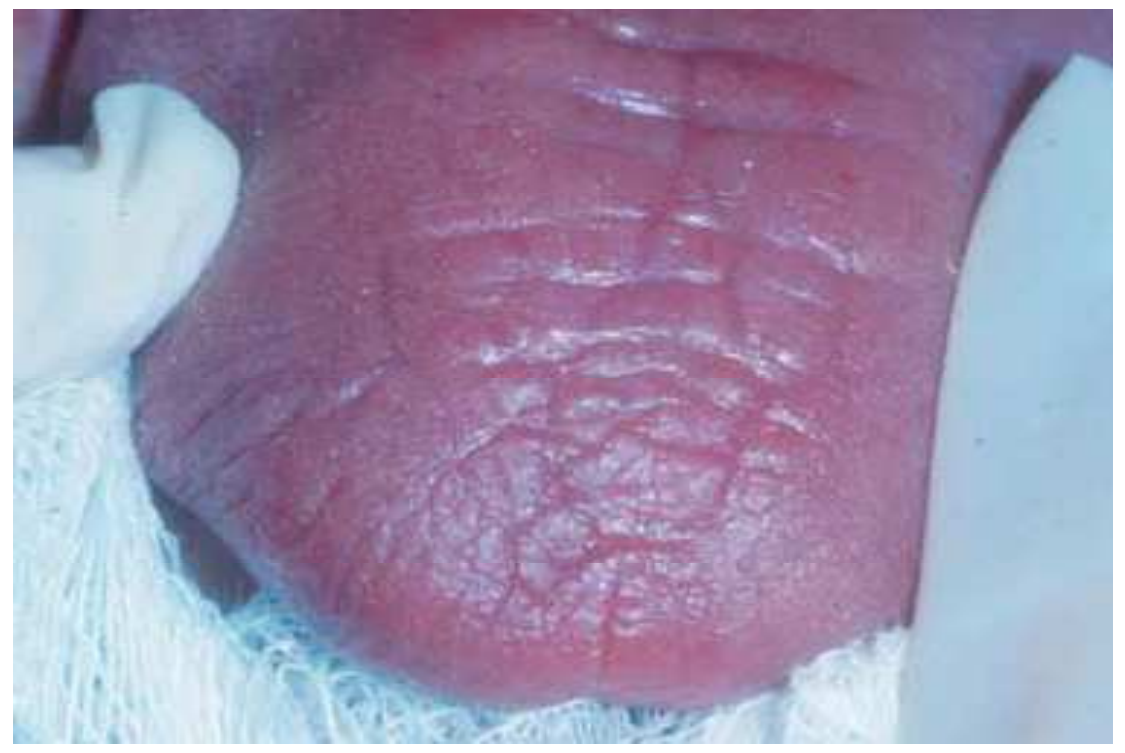

Figure1. Dry mouth associated with Sjögren's syndrome. The tongue is dry and pale and has lost papillation of the surface.

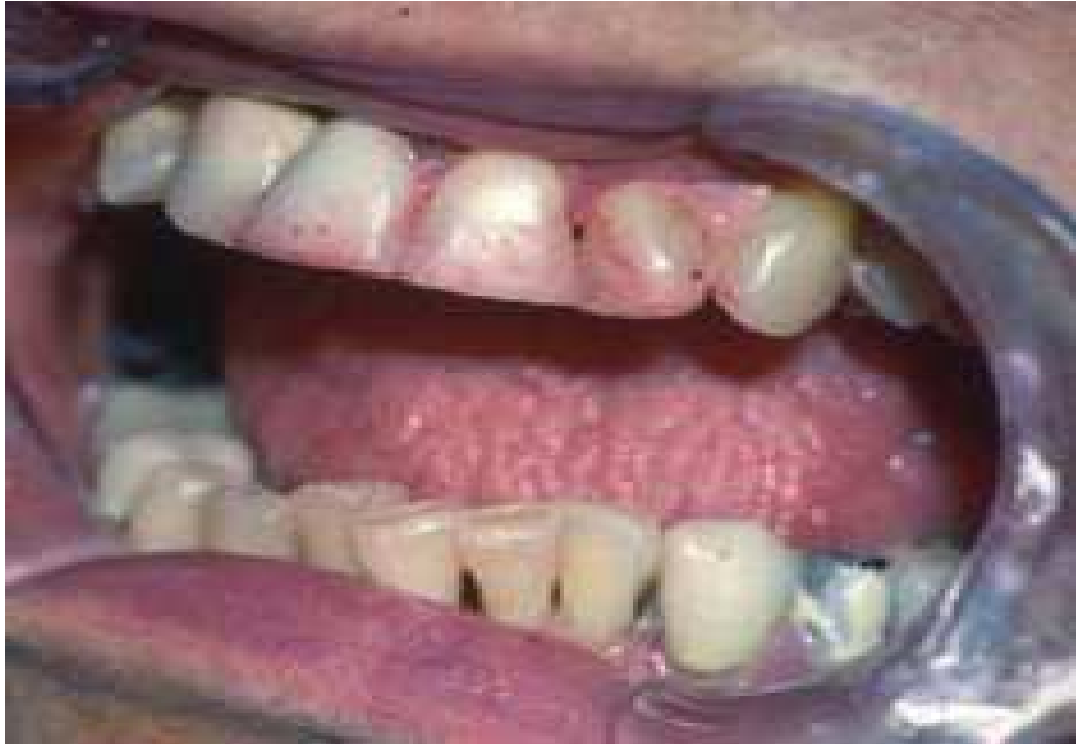

Figure 2. The lips, tongue and all mucosal surfaces are dry in this patient with Sjögren's syndrome. Note also the erosion and the presence of epithelial debris on the teeth, a sign of diminished salivary secretions. 